\title{
Value of splenectomy in patients with Siewert type II adenocarcinoma of the esophagogastric junction
}

\author{
Hironobu Goto $\cdot$ Masanori Tokunaga $\cdot$ Norihiko Sugisawa $\cdot$ Yutaka Tanizawa \\ Etsuro Bando • Taiichi Kawamura • Masahiro Niihara • Yasuhiro Tsubosa • \\ Masanori Terashima
}

Received: 3 September 2012/ Accepted: 2 November 2012/Published online: 18 November 2012

(C) The International Gastric Cancer Association and The Japanese Gastric Cancer Association 2012

\begin{abstract}
Background The incidence of adenocarcinoma of the esophagogastric junction (AEG) has been increasing recently in both Western and Eastern countries. However, an optimal treatment strategy for Siewert type II AEG is still unclear. The aim of this study was to clarify the value of splenectomy in patients with Siewert type II AEG.

Methods From September 2002 to November 2011, 42 patients underwent total gastrectomy with D2 lymph node dissection for Siewert type II AEG and were included in this study. We used the index of estimated benefit from lymph node dissection (IEBLD) to assess the efficacy of lymph node dissection of each station. Surgical complications were graded by the Clavien-Dindo classification.

Results The overall 5-year survival rate of the 42 patients was $57.5 \%$. The incidence of splenic hilar lymph node metastasis was $4.8 \%$ and the 5-year survival rate of patients with splenic hilar lymph node involvement was zero. Consequently, the IEBLD of splenic hilar lymph nodes was zero. Postoperative morbidities occurred in 25 patients $(59.5 \%)$. Pancreas-related complications were the most frequently observed $(28.5 \%)$, followed by intraabdominal abscess $(14.3 \%)$ and anastomotic leakage $(9.5 \%)$.

Conclusions Splenic hilar lymph node dissection may be omitted without decreasing curability in patients with
\end{abstract}

H. Goto $\cdot$ M. Tokunaga $\cdot$ N. Sugisawa $\cdot$ Y. Tanizawa

E. Bando $\cdot$ T. Kawamura $\cdot$ M. Terashima $(\square)$

Division of Gastric Surgery, Shizuoka Cancer Center,

1007 Shimonagakubo, Nagaizumi-cho, Sunto-gun,

Shizuoka 411-8777, Japan

e-mail: m.terashima@scchr.jp

M. Niihara · Y. Tsubosa

Division of Esophageal Surgery, Shizuoka Cancer Center,

Shizuoka, Japan
Siewert type II AEG, although a prospective study is necessary for more conclusive results.

Keywords Gastric cancer - Adenocarcinoma of esophagogastric junction · Siewert type II - Splenectomy

\section{Introduction}

The incidence of adenocarcinoma of the esophagogastric junction (AEG) has been increasing recently in both Western and Eastern countries [1]. In the East, the westernized lifestyle habit and the increased incidence of gastroesophageal reflux disease are thought to be possible reasons, with the incidence of AEG likely to increase further [2]. Siewert et al. [3] classified AEG into three subgroups according to the location of the tumor epicenter. Siewert type I AEG, which is frequently observed in Western countries, is generally treated as an esophageal cancer. Siewert type III AEG, which is frequently observed in Eastern countries, is mostly treated as a gastric cancer. An optimal treatment strategy for Siewert type II AEG is still unclear, and it is under debate whether Siewert type II AEG should be regarded and treated as an esophageal cancer or a gastric cancer $[4,5]$.

The latest European Society for Medical Oncology clinical practice guideline recommends D2 gastrectomy for curable gastric cancer. However, splenectomy is not recommended unless the tumor is directly infiltrating the spleen [6, 7]. In contrast, Japanese guidelines include splenectomy in D2 total gastrectomy. Consequently, splenectomy is mandatory in patients with type II AEG undergoing total gastrectomy in Japan [8,9]. However, recent reports from the East have raised the question of whether splenectomy is valuable in these patients $[10,11]$. 
Reported are an increased incidence of pancreas-related complications following splenectomy and a low incidence of splenic hilar nodal involvement in patients with AEG [10-12]. However, these reports included a variety of patients, such as those with Siewert type I or III AEG and patients undergoing noncurative surgery $[10,11]$; thus, the therapeutic value of splenectomy in patients with Siewert type II AEG undergoing curative gastrectomy remains unclear.

The aim of this study was to clarify the value of splenectomy in patients with Siewert type II AEG. We investigated the clinicopathological characteristics and long-term outcome of patients with Siewert type II AEG who underwent total gastrectomy with D2 lymph node dissection.

\section{Materials and methods}

\section{Patients}

From September 2002 to November 2011, 2,995 patients with gastric cancer underwent gastrectomy at the Shizuoka Cancer Center, Japan. Of these, 64 patients underwent total gastrectomy with D2 lymph node dissection for Siewert type II AEG. Patients with early gastric cancer (13 patients), those who received neoadjuvant chemotherapy (3 patients), and those who underwent noncurative gastrectomy (R1 or R2, 6 patients) were excluded, and the remaining 42 patients were included in the present study.

The International Union Against Cancer (UICC) TNM staging system for esophageal cancer was used for tumor staging [4]. The lymph node stations were numbered according to the definition of the Japanese Gastric Cancer Association (JGCA) [13].

Tumor histology was evaluated according to the JGCA classification [13]. Well- and moderately differentiated tubular adenocarcinoma and papillary adenocarcinoma were classified as differentiated-type carcinomas. Poorly differentiated adenocarcinoma, signet-ring cell carcinoma, and mucinous carcinoma were classified as undifferentiated-type carcinomas.

Patient characteristics and pathological and surgical findings were collected from our database records and individual patient electronic medical records. The data collection and analysis were approved by the institutional review board of the Shizuoka Cancer Center.

\section{Surgery}

Total gastrectomy with D2 lymphadenectomy was carried out in all patients included in the present study. All perigastric nodes and extraperigastric nodes, defined as secondcompartment lymph nodes according to the JGCA classification, were retrieved (2nd English edition of JGCA). To completely remove the splenic hilar lymph nodes, all patients underwent splenectomy. The surgical complications were assessed by the Clavien-Dindo classification [14]. We defined any complication categorized as grade II or higher as a postoperative morbidity.

Evaluation of the therapeutic value of intraabdominal lymph node dissection

In the present study, we adopted the index of estimated benefit from lymph node dissection (IEBLD), a concept proposed by Sasako et al. [15] to assess the efficacy of lymph node dissection of each station. This index is calculated by multiplying the frequency of lymph node metastasis to each station by the 5-year survival rate of patients with positive lymph nodes at each station. The incidence of metastasis and the 5-year survival rate of patients with positive nodes were calculated independently for each lymph node, without any reference to the overall pathological nodal stage.

\section{Statistics}

Statistical analysis was carried out using SPSS version 19 for Windows. The Kaplan-Meier method was used to estimate survival curves. All continuous variables are presented as the median (range).

\section{Results}

\section{Patient characteristics}

The characteristics of the patients are described in Table 1. There were 26 male patients (62\%) and 16 female patients (38\%). Type 3 tumor was the most frequently observed macroscopic type (17 patients, $40.5 \%$ ). The transabdominal approach was the most preferred surgical approach used in this study (37 patients, $88.1 \%$ ). The reconstruction was performed by Roux-en-Y in all cases, and esophagojejunostomy was performed using a circular stapler. Lymph node metastases were observed in 32 patients (76.2 \%: N1, 11 patients; N2, 7 patients; N3, 14 patients). Consequently, tumor stage was determined as IB in 6 patients, IIA in 4 , IIB in 2, IIIA in 8 , IIIB in 3 , and IIIC in the remaining 19 patients. Adjuvant chemotherapy by S-1 was given to 15 patients.

\section{Postoperative morbidities}

The details of postoperative morbidities are described in Table 2. Grade II or higher postoperative complications 
Table 1 Demographics of 42 patients with Siewert type II adenocarcinoma of the esophagogastric junction

\begin{tabular}{|c|c|}
\hline Parameters & $N$ \\
\hline Age median (range), years & $67(30-79)$ \\
\hline \multicolumn{2}{|l|}{ Sex } \\
\hline Male & 26 \\
\hline Female & 16 \\
\hline Tumor size median (range), $\mathrm{mm}$ & $57(20-145)$ \\
\hline \multicolumn{2}{|l|}{ Macroscopic type } \\
\hline Type 0 & 9 \\
\hline Type 1 & 5 \\
\hline Type 2 & 11 \\
\hline Type 3 & 17 \\
\hline \multicolumn{2}{|l|}{ Circumferential distribution } \\
\hline Lesser curvature & 20 \\
\hline Greater curvature & 1 \\
\hline Anterior wall & 4 \\
\hline Posterior wall & 8 \\
\hline Circular & 9 \\
\hline \multicolumn{2}{|l|}{ Histological type } \\
\hline Differentiated & 21 \\
\hline Undifferentiated & 21 \\
\hline \multicolumn{2}{|l|}{ Type of surgery } \\
\hline $\mathrm{TG}+\mathrm{S}$ & 40 \\
\hline $\mathrm{TG}+\mathrm{PS}$ & 2 \\
\hline \multicolumn{2}{|l|}{ Approach } \\
\hline Abdominal & 37 \\
\hline Left thoracoabdominal & 5 \\
\hline \multicolumn{2}{|l|}{ Tumor depth (histological) } \\
\hline $\mathrm{MP}(\mathrm{T} 2)$ & 8 \\
\hline SS(T3) & 22 \\
\hline $\mathrm{SE}(\mathrm{T} 4)$ & 12 \\
\hline \multicolumn{2}{|l|}{ Node stage (histological) } \\
\hline No & 10 \\
\hline N1 & 11 \\
\hline $\mathrm{N} 2$ & 7 \\
\hline N3 & 14 \\
\hline \multicolumn{2}{|l|}{ Stage } \\
\hline IA & 0 \\
\hline IB & 6 \\
\hline IIA & 4 \\
\hline IIB & 2 \\
\hline IIIA & 8 \\
\hline IIIB & 3 \\
\hline IIIC & 19 \\
\hline IV & 0 \\
\hline \multicolumn{2}{|l|}{ Adjuvant chemotherapy (S-1) } \\
\hline+ & 15 \\
\hline- & 27 \\
\hline
\end{tabular}

$P S$ pancreaticosplenectomy, $S$ splenectomy, $T G$ total gastrectomy, $M P$ muscularis propria, $S S$ subserosa, $S E$ exposed beyond the serosa
Table 2 Postoperative complications in 42 patients after total gastrectomy with D2 lymphadenectomy

\begin{tabular}{lrr}
\hline Complications & $n$ & $\%$ \\
\hline Complication, grade II or higher $^{\mathrm{a}}$ & 25 & 59.5 \\
Pancreas-related complication & 12 & 28.5 \\
Intraabdominal abscess & 6 & 14.3 \\
Anastomotic leakage & 4 & 9.5 \\
Pneumonia & 4 & 9.5 \\
Pleural fluid & 4 & 9.5 \\
Bleeding & 2 & 4.8 \\
Cholecystitis & 1 & 2.4 \\
Wound complication & 1 & 2.4 \\
\hline
\end{tabular}

${ }^{\text {a }}$ Based on the Clavien-Dindo classification [14]

occurred in 25 patients (59.5\%). Pancreas-related complications were the most frequently observed morbidity (28.5\%), followed by intraabdominal abscess (14.3\%) and anastomotic leakage $(9.5 \%)$.

Survival outcomes

The 5-year survival rate of the 42 patients in this study was $57.5 \%$. Table 3 presents the frequency of metastasis of each regional lymph node, the 5-year survival rate of patients with nodal involvement, and the IEBLD for each station.

Lymph node involvement was observed in more than $10 \%$ of patients (range 16.7-59.5\%) in stations 1, 2, 3, 7, 9 , and $11 \mathrm{p}$, and the IEBLDs of these stations ranged from 5.6 to 30.3 . The incidence of metastasis was lower than $10 \%$ (range $0-9.5 \%$ ) in the other stations, and the IEBLD was low $(0-4.8)$. Lymph node metastasis was not found in stations $4 \mathrm{~d}$ and $12 \mathrm{a}$. In addition, the 5-year survival rate was zero if station $4 \mathrm{sa}, 4 \mathrm{~d}, 6,8 \mathrm{a}, 10,11 \mathrm{~d}$, or $12 \mathrm{a}$ was involved.

Consequently, the IEBLDs of stations that were located far from the esophagogastric junction, such as stations $4 \mathrm{~d}$, 6 (along the right gastroepiploic artery), 8a (along the common hepatic artery), and 12a (along the proper hepatic artery), were zero. In addition, the IEBLDs of stations 10 and $11 \mathrm{~d}$, where splenectomy is necessary for complete retrieval of these nodes, were also zero (Fig. 1).

\section{Discussion}

The present study shows the IEBLD of regional lymph nodes in patients with Siewert type II AEG ranged from 0 to 30.3. It is plausible that dissection of some of these stations could be omitted even in advanced cases.

In the present study, the IEBLD of stations 1, 2, 3, 7, 9, and $11 \mathrm{p}$ were higher than the other stations. Previous 
Table 3 Frequency of lymph node metastasis and 5-year survival for each lymph node station
$I E B L D$ index of estimated benefit from lymph node dissection

\begin{tabular}{llllll}
\hline $\begin{array}{l}\text { Lymph } \\
\text { node } \\
\text { station }\end{array}$ & $\begin{array}{l}\text { Number of } \\
\text { patients with } \\
\text { metastatic nodes }\end{array}$ & $\begin{array}{l}\text { Number of patients } \\
\text { in whom the station } \\
\text { was dissected }\end{array}$ & $\begin{array}{l}\text { Incidence of } \\
\text { lymph node } \\
\text { metastasis }(\%)\end{array}$ & $\begin{array}{l}\text { Five-year survival rate } \\
\text { of patients with } \\
\text { metastatic nodes }(\%)\end{array}$ & IEBLD \\
\hline 1 & 25 & 42 & 59.5 & 50.3 & 29.9 \\
2 & 8 & 42 & 19.0 & 46.9 & 8.9 \\
3 & 24 & 42 & 57.1 & 53.0 & 30.3 \\
$4 \mathrm{sa}$ & 1 & 42 & 2.4 & 0 & 0 \\
$4 \mathrm{sb}$ & 2 & 42 & 4.8 & 50.0 & 2.4 \\
$4 \mathrm{~d}$ & 0 & 42 & 0 & 0 & 0 \\
5 & 1 & 42 & 2.4 & 100 & 2.4 \\
6 & 1 & 42 & 2.4 & 0 & 0 \\
7 & 13 & 42 & 30.9 & 58.4 & 18.1 \\
$8 \mathrm{a}$ & 1 & 2.4 & 0 & 0 \\
9 & 10 & 42 & 23.8 & 30.0 & 7.1 \\
10 & 2 & 42 & 4.8 & 0 & 0 \\
$11 \mathrm{p}$ & 7 & 42 & 16.7 & 33.3 & 5.6 \\
$11 \mathrm{~d}$ & 2 & 42 & 4.8 & 0 & 0 \\
$12 \mathrm{a}$ & 0 & 42 & 0 & 0 & 0 \\
19 & 4 & 14 & 9.5 & 50.0 & 4.8 \\
20 & 2 & 42 & 4.8 & 50.0 & 2.4 \\
\hline
\end{tabular}

studies also reported a high IEBLD in these stations in patients with AEG $[10,11]$. Investigation of lymphatic flow showed that these stations were sentinel nodes for the upper part of the stomach [16]. We consider complete retrieval of these stations would be of value and should not be omitted during curative surgery for Siewert type II AEG.

The IEBLDs of the remaining stations were lower than that of station $1,2,3,7,9$, and $11 \mathrm{p}$, and was zero in stations 4sa, 4d, 6, 8a, 10, and 11d; thus, lymph node dissection of some of these stations could be omitted. If we omit the supra- and infrapyloric lymph nodes, then the distal part of the stomach might be preserved [17]. In addition, the spleen could be preserved if the station 10 lymph node dissection was omitted, even in cases with advanced disease.

Previously, the therapeutic value of removing station 10 lymph nodes in AEG was investigated, and similar results, a low IEBLD for station 10 (0-2.2), were reported [5, 10, 11]. Yamashita et al. investigated IEBLD of 225 patients with Siewert type II AEG, and reported that of station 10 was 0.7. In addition, Hosokawa et al. reported IEBLD for station 10 in patients with AEG was 2.2. However, these previous studies included patients underwent non-curative gastrectomy or those with Siewert type I or III AEG. Therefore, to the best of our knowledge, our study is the first study to investigate the IEBLD of station 10 in patients with Siewert type II AEG underwent curative surgery.

The circumferential distribution is a possible reason why IEBLD of station 10 was zero in this study. Of 42 patients with circumferentially localized AEGs, the AEG was located along the lesser curvature in 20 patients. In contrast, it was located along the greater curvature in only one patient, the resulting tumor location being far away from the spleen, and there was a low incidence of station 10 lymph node involvement in this series. The same trend was also reported by Suh et al. [5] previously.

It would be advantageous to avoid splenectomy, a procedure necessary for the complete removal of the splenic hilar lymph node, as it has been reported that splenectomy increases pancreas-related complications and disturbs immune functions [12, 18, 19]. In fact, pancreasrelated complications were the most frequently observed complication $(28.5 \%)$ in our study, and it was higher than that after spleen-preserving total gastrectomy in our institute (2.5\%; data not shown). Even in Japan, where splenectomy is mandatory for D2 gastrectomy, some studies have reported a lack of survival benefit and increased postoperative morbidities in patients undergoing splenectomy [12, 20-22]. In the West, although the current standard treatment for curable gastric cancer is a D2 gastrectomy, splenectomy is not a mandatory procedure, presumably because of increased postoperative pancreas-related complications and a lack of evidence that supports the feasibility of splenectomy [23-26]. Thus, splenectomy itself increases postoperative morbidities. Therefore, it would be of value if we could avoid splenectomy without worsening the long-term outcome of patients. 

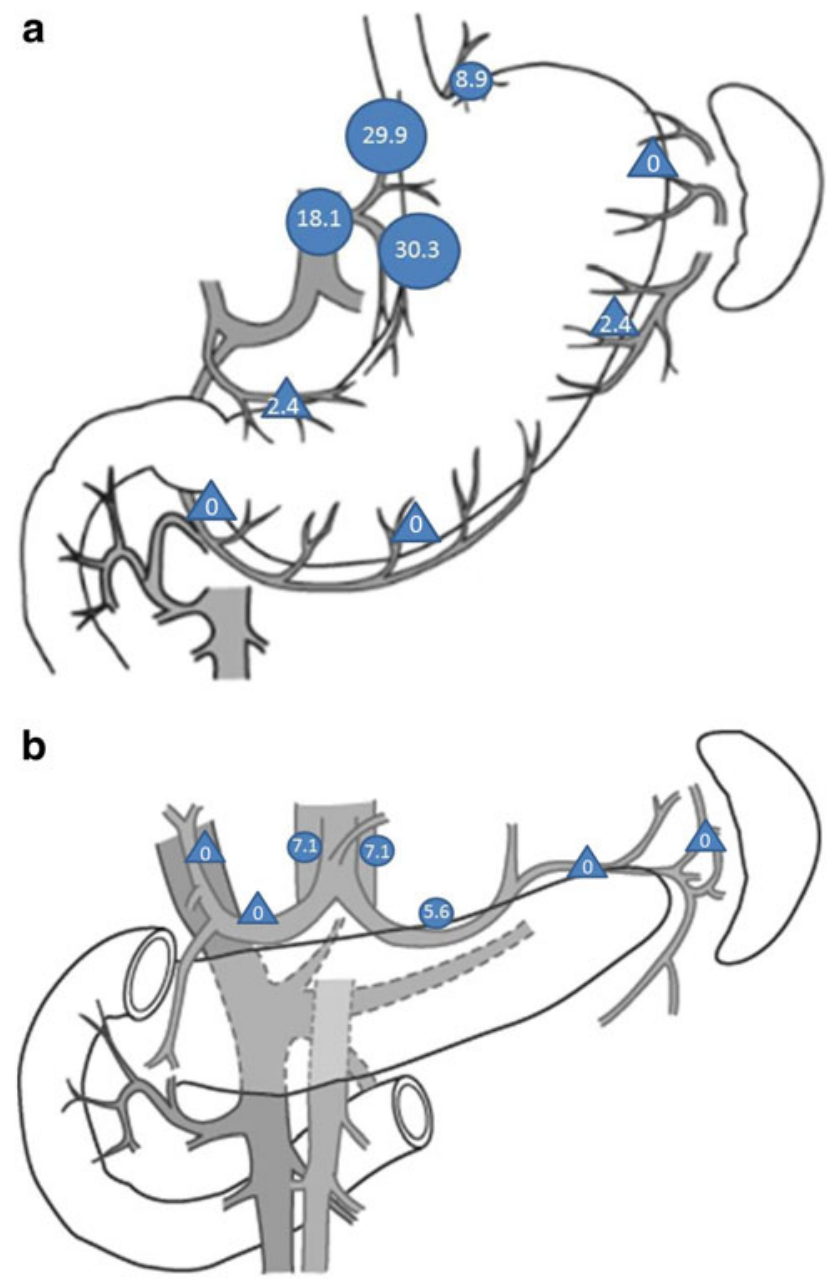

Fig. 1 Index of estimated benefit from lymph node dissection (IEBLD) for each lymph node station. Circles, IEBLD greater than 5 ; triangles, IEBLD less than 5

The present retrospective study has limitations, which include the small number of patients investigated in the study. Even though the number of patients with Siewert type II AEG has been increasing in Japan, the number of patients at each institute is still limited. Second, mediastinal lymph node dissection was not performed routinely, particularly in the early period of this study. Consequently, the value of mediastinal lymph node dissection cannot be assessed. The latest JGCA guidelines recommend mediastinal lymph node dissection for patients with AEG; thus, we currently perform lower mediastinal lymph node dissection for these patients (14th JGCA guidelines). We will be able to assess the value of lower mediastinal lymph node dissection in the near future.

In conclusion, the IEBLD of the splenic hilar lymph nodes was zero in the present study. Splenic hilar lymph node dissection may be omitted without decreasing curability in patients with Siewert type II AEG, although a prospective study is necessary for more conclusive results.

\section{References}

1. Oda I, Abe S, Kusano C, et al. Correlation between endoscopic macroscopic type and invasion depth for early esophagogastric junction adenocarcinomas. Gastric Cancer. 2011;14:22-7.

2. Kusano C, Gotoda T, Khor CJ, et al. Changing trends in the proportion of adenocarcinoma of the esophagogastric junction in a large tertiary referral center in Japan. J Gastroenterol Hepatol. 2008;11:1662-5.

3. Siewert JR, Feith M, Werner M, et al. Adenocarcinoma of the esophagogastric junction: results of surgical therapy based on anatomical/topographic classification in 1002 consecutive patients. Ann Surg. 2000;232:353-61.

4. Biondi A, Hyung WJ. Seventh edition of TNM classification for gastric cancer. J Clin Oncol. 2011;29:4338-9.

5. Suh YS, Han DS, Kong SH, et al. Should adenocarcinoma of the esophagogastric junction be classified as esophageal cancer? A comparative analysis according to the seventh AJCC TNM classification. Ann Surg. 2012;255:908-15.

6. Okines A, Verheij M, Allum W, et al. Gastric cancer: ESMO clinical practice guidelines for diagnosis, treatment and follow up. Ann Oncol. 2010;21(suppl 5):v50-4.

7. Hartgrink H, Jansen E, Grieken N, et al. Gastric cancer. Lancet. 2009;374:477-90.

8. Japanese Gastric Cancer Association. Japanese gastric cancer treatment guidelines 2010 (ver.3). Gastric Cancer. 2010;14:113-23.

9. Katai H, Yoshimura K, Fukagawa $T$, et al. Risk factors for pancreas-related abscess after total gastrectomy. Gastric Cancer. 2005;8:137-41.

10. Yamashita H, Katai H, Morita S, et al. Optimal extent of lymph node dissection for Siewert type II esophagogastric junction carcinoma. Ann Surg. 2011;254:274-80.

11. Hosokawa Y, Kinoshita T, Konishi M, et al. Clinicopathological features and prognostic factors of adenocarcinoma of the esophagogastric junction according to Siewert classification: experiences at a single institution in Japan. Ann Surg Oncol. 2012;19:677-83.

12. Sano T, Sasako M, Shibata T, et al. Randomized controlled trial to evaluate splenectomy in total gastrectomy for proximal gastric carcinoma (JCOG0110): analyses of operative morbidity, operation time, and blood loss. J Clin Oncol. 2010;28(15 suppl):4020. (ASCO meeting abstract).

13. Japanese Gastric Cancer Association. Japanese classification of gastric carcinoma: 3rd English edition. Gastric Cancer. 2011;14: 101-12.

14. Clavien PA, Barkun J, de Oliveira ML, et al. The Clavien-Dindo classification of surgical complications. Ann Surg. 2009;250: 187-96.

15. Sasako M, McCulloch P, Kinoshita T, et al. New method to evaluate the therapeutic value of lymph-node dissection for gastric cancer. Br J Surg. 1995;82:346-51.

16. Miwa K, Kinami S, Taniguchi K, et al. Mapping sentinel nodes in patients with early-stage gastric carcinoma. Br J Surg. 2003;90: 178-82.

17. Kobayashi T, Sugimura H, Kimura T. Total gastrectomy is not necessary for advanced gastric cancer of the cardia. Dig Surg. 2002;19:15-21.

18. Cuschieri A, Weeden S, Fielding J. Patient survival after D1 and D2 resections for gastric cancer: long-term results of the MRC randomized surgical trial. Br J Cancer. 1999;79:1522-30.

19. Ram S, Lewis LA, Rice PA. Infections of people with complement deficiencies and patients who have undergone splenectomy. Clin Microbiol Rev. 2010;23:740-80.

20. Kodera Y, Yamamura Y, Shimizu Y, et al. Lack of benefit of combined pancreaticosplenectomy in D2 resection for proximalthird gastric carcinoma. World J Surg. 1997;21:622-8. 
21. Maehara Y, Moriguchi S, Yoshida M, et al. Splenectomy does not correlate with length of survival in patients undergoing curative total gastrectomy for gastric cancer. Cancer (Phila). 1991;67: 3006-9.

22. Kitamura K, Nishida S, Ichikawa D, et al. No survival benefit from combined pancreaticosplenectomy and total gastrectomy for gastric cancer. Br J Surg. 1999;86:119-22.

23. Csendes A, Burdiles $\mathrm{P}$, Rojas $\mathrm{J}$, et al. A prospective randomized study comparing D2 total gastrectomy versus D2 gastrectomy plus splenectomy in 187 patients with gastric cancer. Surgery (St. Louis). 2002;131:401-7.
24. Hartgrink H, Velde C, Putter H, et al. Extended lymph node dissection for gastric cancer: who may benefit? Final results of the randomized Dutch Gastric Cancer Group Trial. J Clin Oncol. 2004;22:2069-77.

25. Brady MS, Rogatko A, Dent LL, et al. Effect of splenectomy on morbidity and survival following curative gastrectomy for carcinoma. Arch Surg. 1991;126:359-64.

26. Wanebo HJ, Kennedy BJ, Winchester DP, et al. Role of splenectomy in gastric cancer surgery: adverse effect of elective splenectomy on longterm survival. J Am Coll Surg. 1997;185:177-84. 